similar case reports and linear studies would help us to forecast clinical prognosis in these cases.

\section{G30(P) ATYPICAL PRESENTATION OF ARVC MIMICKING ACUTE CORONARY SYNDROME}

${ }^{1}$ AA Bapputty Haji, ${ }^{2} \mathrm{C}$ Gonzalez Corcia, ${ }^{2}$ AJ Matthews, ${ }^{3}$ A Wong, ${ }^{3} \mathrm{O}$ Uzun. ${ }^{1}$ Paediatrics, Birmingham Heartlands Hospital, Birmingham, UK; ${ }^{2}$ Paediatric Cardiology, Bristol Royal Children's Hospital, Bristol, UK; ${ }^{3}$ Paediatric Cardiology, University Hospital of Wales, Cardiff, UK

\subsection{6/archdischild-2020-rcpch.18}

Introduction Arrhythmogenic Right Ventricular Cardiomyopathy (ARVC) is an autosomal dominant inherited disease of the heart muscle. It is characterised by replacement of cardiomyocytes with fibro-fatty tissue, predominantly affecting the right ventricle. This process leads to thinning of the ventricular wall and progression to heart failure. However, its major immediate complications are the increased risk of ventricular arrhythmia and sudden cardiac death (SCD).

Case Report We report a 15-year-old, who was under surveillance due to a family history of suspected ARVC in his mother, presented with excruciating chest pain which started while watching a football match. Significantly raised troponin-I level of $>3000$ (later found to be due to auto-antibodies), and a CK of 175 prompted to initially treat him for suspected acute coronary syndrome. Subsequent ECGs showed epsilon waves and inverted $\mathrm{T}$ waves in leads V1-V5, whereas echocardiography and cardiac CT angiogram were found normal. But cardiac MRI demonstrated fatty infiltration in the inferolateral wall of the RV and LV myocardium, along with mild RV dilation and dysfunction. Hence an early and severe onset of ARVC was confirmed according to Task Force Major Criteria of 2010. The presence of polymorphic ventricular ectopics along with short runs of 4 beats of nonsustained VT resulted in an indication for an early ICD implantation.

Discussion This is a rare case of ARVC that mimics acute coronary syndrome, due to a severe onset of fatty myocardium infiltration resulting in myocardial cell injury and elevated troponin levels. ARVC is generally asymptomatic, being diagnosed in young males (especially athletes) who present with ventricular arrhythmias, syncope or SCD. Diagnosis of ARVC is very difficult due to its variable penetration and incomplete expression.

Conclusion Chest pain with high elevation of troponin level is a rare presentation of ARVC. We highlight the importance of considering ARVC as a differential diagnosis in young patients presenting with chest pain and elevated troponin, especially in the presence of ECG findings of epsilon waves or repolarisation abnormalities in the right precordial leads. An early diagnosis is crucial in this disease to implement strategies to prevent and/or treat ventricular arrhythmias and SCD, including lifestyle restrictions and placement of an ICD.

\section{G31(P) ABSTRACT WITHDRAWN}

British academy of childhood disability and british paediatric neurology association

\section{Plenary presentation}

\section{P08 EPIDEMIOLOGY OF EPILEPSY IN EARLY CHILDHOOD AND PREDICTORS OF OUTCOME: A NATIONAL POPULATION-BASED PROSPECTIVE COHORT STUDY}

${ }^{1,2} \mathrm{JD}$ Symonds, ${ }^{3} \mathrm{KS}$ Elliott, ${ }^{3} \mathrm{JC}$ Knight, ${ }^{4} \mathrm{~A}$ Jollands, ${ }^{4} \mathrm{E}$ Pilley, ${ }^{5}$ J Shetty, ${ }^{6} \mathrm{E}$ Stephen,

${ }^{1,2}$ SM Zuberi. 'Paediatric Neurosciences Research Group, Royal Hospital for Children, Glasgow, UK; ${ }^{2}$ Medical Veterinary and Life Sciences, University of Glasgow, Glasgow, UK; ${ }^{3}$ Wellcome Trust Centre for Human Genetics, University of Oxford, Oxford, UK; ${ }^{4}$ Paediatric Neurology, Tayside Children's Hospital, Dundee, UK; ${ }^{5}$ Paediatric Neurology, Royal Hospital of Sick Children, Edinburgh, UK; ${ }^{6}$ Paediatric Neurology, Royal Aberdeen Children's Hospital, Aberdeen, UK

\subsection{6/archdischild-2020-rcpch. 19}

\section{Objectives}

1. To calculate the incidence of early childhood-onset epilepsy

2. To report the yield from aetiological investigations in these patients

3. To investigate for predictors of Drug Resistant Epilepsy (DRE) and Global Developmental Delay (GDD) at 24 months after presentation

Methods This was a prospective national cohort study involving all paediatric centres in Scotland. Patients presenting with epilepsy before their 3rd birthday were identified over a 3 year period (May 8th 2014 to May 7th 2017). Two independent sources were used: 1 . A prospective nationwide case identification study; 2. Retrospective case note review of all children attending for EEG investigation.

Capture-recapture analysis was used to estimate missing cases. Clinical follow-up data were obtained 24 months after first presentation with seizures, including presence of DRE and/or GDD. Logistic regression was used to identify predictors of DRE and GDD.

Patients all had brain MRI and received genetic testing (104 gene epilepsy panel and chromosomal microarray). Those negative on gene panel and microarray underwent Whole Genome Sequencing.

Results Population statistics for Scotland were: Total $<3$ years population 169,500; mean births per year (2011-2016) 56,490

390 patients with epilepsy were identified. Using capturerecapture the estimated total was 406 . The incidence of epilepsy <3 years was 1 per 417 live births (95\% CI 377463).

211 (54\%) patients had an aetiology identified, of which 72 were primarily structural and 133 were primarily genetic. Of the genetic diagnoses 7 were trisomies, 24 were chromosomal microdeletions/duplications, and 102 were single gene variants.

At 24 month follow-up 139 patients (36\%) had DRE, and 193 (49\%) had GDD. Early age of presentation $(<12$ months) and identification of aetiology were associated with DRE. Initial seizure type (focal seizures or epileptic spasms) and identification of aetiology were associated with GDD (all $\mathrm{p}<0.05)$.

Conclusion One in 400 children develops epilepsy before their third birthday. The previous estimate in this age group from 
Rochester Minnesota was 1 per 614. One third of these children develops DRE and half have GDD. Aetiology can be identified in 54\%. Identification of aetiology is associated with DRE and GDD.

\section{Plenary}

\section{P07 ONASEMNOGENE ABEPARVOVEC GENE-REPLACEMENT THERAPY (GRT) FOR SPINAL MUSCULAR ATROPHY (SMA): FROM BENCH TO BEDSIDE}

${ }^{1} \mathrm{P}$ Kaufmann, ${ }^{1} \mathrm{~K}$ Foust, ${ }^{1} \mathrm{~J}$ Loukes, ${ }^{2,3,4} \mathrm{~J}$ Mendell. ${ }^{1}$ AveXis, Inc., Bannockburn, IL, United States; ${ }^{2}$ Center for Gene Therapy, Nationwide Children's Hospital, Columbus, $\mathrm{OH}$, United States; ${ }^{3}$ Department of Pediatrics, Ohio State University, Columbus, OH, United States; ${ }^{4}$ Department of Neurology, Ohio State University, Columbus, $\mathrm{OH}$, United States

\subsection{6/archdischild-2020-rcpch.20}

Aims Report onasemnogene abeparvovec (formerly AVXS101) GRT development for SMA. Onasemnogene abeparvovec is a one-time, intravenous GRT that addresses the genetic root cause of SMA, a progressive neurological disease. Onasemnogene abeparvovec delivers the survival motor neuron gene $(S M N)$ via a self-complementary adeno-associated serotype 9 viral vector (scAAV9) that crosses the blood-brain barrier. Onasemnogene abeparvovec is designed for immediate and sustained expression of SMN protein in non-dividing neurons, allowing for rapid onset and durable therapeutic effect.

Methods SMA mice $\left(S M N 2^{+/+} ; S M N \Delta 7^{+/+} ; \mathrm{Smn}^{-/}\right)$received intravenous scAAV9-SMN or scAAV9-GFP at post-natal day 1; survival and motor function were assessed. Non-human primates (NHPs) received intravenous scAAV9-GFP; transduced cell types were assessed. A phase $1 / 2 \mathrm{a}$ study (START; NCT02122952) evaluated onasemnogene abeparvovec infused at low (Cohort $1, n=3$ ) or high (Cohort 2, $n=12$ ) doses in symptomatic SMA type 1 (SMA1) infants; patients were followed for 2 years for safety/tolerability and efficacy and could enroll in a long-term follow-up (LTFU) study to assess longterm safety.

Results In SMA mice, scAAV9-SMN improved survival (>200 versus 15 days in controls), and increased motor function. In NHPs, scAAV9-GFP efficiently targeted motor neurons throughout the central nervous system. In the phase $1 / 2 \mathrm{a}$ START trial, all patients survived free of permanent ventilation at 24 months. In the high-dose cohort, 11/12 patients reached CHOP INTEND $>40 ; 11$ sat unassisted $\geq 5 \mathrm{~s}, 10$ for $\geq 10$ s, 9 for $\geq 30$ s. Two patients crawled, stood, and walked. No patient received nusinersen during the 24-month study period. In the LTFU study, 2 more patients in Cohort 2 have gained the motor milestone of standing with assistance (as of 31 Dec 2019); neither patient has ever received nusinersen. No new treatment-related serious adverse events or adverse events of special interest have occurred in LTFU (as of 31 Dec 2019). The oldest patient in Cohort 2 is aged 5.6 years, with 5.2 years of follow-up since onasemnogene abeparvovec dosing.

Conclusion Onasemnogene abeparvovec has demonstrated unprecedented outcomes in patients with SMA compared with untreated natural history. Phase 3 trials of onasemnogene abeparvovec in SMA1 are ongoing (US, EU, Asia-Pacific); additional trials are investigating presymptomatic SMA and intrathecal administration in older patients.

\section{G32 IMPROVING SURVIVAL OF THOSE WITH CEREBRAL PALSY IN ENGLAND AND WALES OVER THE LAST 18 YEARS}

JRF Sanner, MN Hurley. Nottingham Children's Hospital, Queen's Medical Centre, Nottingham, UK

\subsection{6/archdischild-2020-rcpch.21}

Aims To assess the rate of change in median age of death over time, where cerebral palsy (CP) is documented as a cause of death, with reference to all-cause mortality in England and Wales.

Methods Published death registration statistics for England and Wales for all-cause mortality as well as ICD 10 code 'G80 Cerebral Palsy' were extracted from the WHO Causes of Death Query online for 2001-2016 and the Office for National Statistics for 2013-2018. This captures those who died, where cerebral palsy was documented in part 1 of the death certificate. Linear interpolation was used to calculate the median age of death for the different groups. Data was imported into SPSS and linear regression was used to assess trends.

Results Median age of death was significantly lower in the CP group compared to the general population, with the median age of death increasing from 22.5 in 2001 to 49.6 in 2018. The trends in median age of death from 2001-2018 in England and Wales are highlighted in table 1.

Abstract G32 Table 1 Change in median age of death (in life years per year) from 2001-2018 in England and Wales

\begin{tabular}{lll}
\hline & Cerebral Palsy & All Causes \\
\hline Combined & $+1.59(95 \% \mathrm{Cl} 1.19$ to 1.99$)$ & $+0.15(95 \% \mathrm{Cl} 0.11$ to 0.18$)$ \\
Male & $+1.61(95 \% \mathrm{Cl} 1.23$ to 1.99$)$ & $+0.14(95 \% \mathrm{Cl} 0.13$ to 0.15$)$ \\
Female & $+1.46(95 \% \mathrm{Cl} 0.88$ to 2.04$)$ & $+0.11(95 \% \mathrm{Cl} 0.10$ to 0.12$)$ \\
\hline
\end{tabular}

Conclusion The increasing median age of deaths due to CP hints at improved survival due to improvements in care. The rate of this improvement is significantly greater than that of the general population.

These death registration data cannot directly report improved survival. However, this finding is supported by the absolute numbers for registration of deaths due to $\mathrm{CP}$ remaining constant. Prolonged survival may therefore be inferred by the increasing median age of death.

\section{G33 EACH AND EVERY NEED - A COMPLETED AUDIT CYCLE OF ACUTE CARE RECEIVED BY CHILDREN WITH CHRONIC NEURODISABILITY}

S Steadman, M Sawal. Community Paediatrics, Royal Wolverhampton NHS Trust, Wolverhampton, UK

\subsection{6/archdischild-2020-rcpch.22}

Aims In 2018 the National Confidential Enquiry into Patient Outcome and Death (NCEPOD) launched 'Each and Every Need: a review of the quality of care provided to patients 0 25 with chronic neurodisability' detailing the standards of care relating to both acute and community care. Our experience was that these were not being met when our complex 